\title{
RIGIDITY THEOREMS FOR RIGHT ANGLED REFLECTION GROUPS
}

\author{
ENNIS ROSAS
}

\begin{abstract}
Let $\Gamma$ be a right angled reflection group. Let $M$ and $M^{\prime}$ be Coxeter manifolds. Then any $\Gamma$-map $f: M \rightarrow M^{\prime}$ is $\Gamma$-homotopic to a homeomorphism
\end{abstract}

Introduction. This paper contains the results given in the author's Ph.D. thesis.

Davis in [9], showed that in every dimension $\geq 4$, there exists a cocompact reflection group on a contractible manifold not homeomorphic to an Euclidean space. Also he gave the first example of a closed aspherical manifold not covered by Euclidean space.

Davis construction provides an infinite Coxeter group acting locally smoothly, effectively and properly discontinuously on a contractible manifold with compact quotient.

The construction is as follows: Let $K$ be a finite simplicial complex that is a generalized homology sphere. Let $V$ be the set of vertices of $K^{\prime}$ (the baricentric subdivision of $K)$.

So $V=\left\{\sigma^{\prime} \mid \sigma\right.$ is a simplex of $\left.K\right\}$. Define $m: V \times V \rightarrow\{1,2,3,4, \ldots, \infty\}$ by $m\left(\sigma^{\prime}, \tau^{\prime}\right)=1$ if $\sigma=\tau, 2$ if $\sigma<\tau$ or $\tau<\sigma$, and $\infty$ otherwise. This defines a Coxeter group $(\Gamma, V)$ (see 1.1).

Let $K^{\prime \prime}$ be the dual polyhedron of $K$. There is a recognized fact in surgery theory that there exists a contractible compact manifold $X^{n}, n \geq 4$, such that $\partial X=K^{\prime \prime}$. Give to $X$ the following $V$-panelled structure $\left(X_{\sigma^{\prime}}\right)_{\sigma^{\prime} \in V}$, where $X_{\sigma^{\prime}}$ is defined as the dual cell of $\sigma^{\prime}$. So $X_{S}$ is $\varnothing$ if $S=\left\{\sigma_{1}^{\prime}, \sigma_{2}^{\prime}, \sigma_{k}^{\prime}\right\}$ is not a simplex of $K^{\prime}$ or the dual $n-k$ cell of $S$ if $S$ is a $k$-simplex. In this form $\left\{X,\left(X_{\sigma^{\prime}}\right)_{\sigma^{\prime} \in V}\right\}$ is a contractible $V$-panelled manifold (see [9]), and each face of $X$ is contractible.

Form the universal space $U(\Gamma, X)$ (see 1.5). By [9, Theorem 10.1], $U(\Gamma, X)$ is contractible, and by $1.12, U(\Gamma, X)$ is a manifold. Also $\Gamma$ acts as cocompact reflection group.

All the Davis examples on which $U(\Gamma, X) \neq R^{n}, n \geq 4$, come from "right angled Coxeter groups" (this means that the only entries of the Coxeter matrix are 1, 2, and $\infty)$.

The construction has the additional attractive property that $U(\Gamma, X)^{H}$ is contractible if $H$ is any finite subgroup of $\Gamma$ (see 2.12).

These $\Gamma$-manifolds $U(\Gamma, X)$ motivate the definition of Coxeter manifolds. A Coxeter manifold is a Coxeter group $\Gamma$ and a manifold $N$ on which $\Gamma$ acts in a locally smooth, effective and properly discontinuous fashion such that $N^{H}$ is contractible

Received by the editors June 17, 1987.

1980 Mathematics Subject (llassification (1985 Revision). Primary 57S25; Secondary 57R99. 
for each finite subgroup $H$ of $\Gamma, N / \Gamma$ is compact and if $H$ is a finite subgroup of $\Gamma$ such that $N^{H}$ has dimension three, then $N^{H} \approx R^{3}$.

The purpose of this thesis is to prove the following conjecture: If $(M, \Gamma)$ and $\left(M^{\prime}, \Gamma\right)$ are Coxeter manifolds, then $M$ and $M^{\prime}$ are equivariantly homeomorphic.

A Coxeter group $(\Gamma, V)$ is said to be a right angled reflection group if $\Gamma$ acts on a contractible manifold $Y^{n}$ with compact quotient and satisfies the following conditions:

(1) The generators $v \in V$ acts as reflections on $Y$.

(2) The action is locally smooth, effective and properly discontinuous.

(3) The only entries of the Coxeter matrix are 1,2 and $\infty$.

(4) If $V \supset S$, such that $S$ generates a finite subgroup $G$ of $\Gamma$, then $Y^{G}$ is contractible.

The main theorem.

THEOREM 3.11 (RIGIDITY THEOREM FOR COXETER MANIFOLDS). Let $(M, \Gamma)$ and $\left(M^{\prime}, \Gamma\right)$ be two Coxeter manifolds. Assume that $\Gamma$ is a right angled reflection group. Then $M$ is equivariantly homeomorphic to $M^{\prime}$. In fact, any $\Gamma$ map $f: M \rightarrow M^{\prime}$ is $\Gamma$-homotopic to a homeomorphism.

There is evidence that the right angled reflection group hypothesis is unnecessary.

The author would like to thank his thesis advisor, Frank Connolly, for his help, suggestions and discussions on the preparation of this paper.

\section{CHAPTER 1. BASIC DEFINITIONS AND THEOREMS}

Coxeter groups. Let $\Gamma$ be a group and $V$ be a set of generators, each element of which has order two. For any pair of elements $\left(s, s^{\prime}\right)$ of $V$, denote by $m\left(s, s^{\prime}\right)$ the order of $s s^{\prime}$, and let $I$ be the set of pairs $\left(s, s^{\prime}\right)$ of distinct elements in $V$ such that $m\left(s, s^{\prime}\right)$ is finite.

DEFINITION $1.1[\mathbf{1}]$. The pair $(\Gamma, V)$ is a Coxeter group if the set of generators $V$ together with the relations

$s^{2}=1, s \in V$,

$\left(s s^{\prime}\right)^{m\left(s, s^{\prime}\right)}=1$, where $\left(s, s^{\prime}\right) \in I$

form a presentation for $\Gamma$.

If the order of $s s^{\prime}$ is two or infinite, for all $\left(s, s^{\prime}\right) \in I$, we say that $(\Gamma, V)$ is a right angled Coxeter group.

Let $(\Gamma, V)$ be a Coxeter group and $V \supseteq S$. Denote by $\Gamma_{s}$ the subgroup of $\Gamma$ generated by $S$.

Proposition 1.2 [1, P. 19]. Let $g \in \Gamma$. There exists a subset $V_{g}$ of $V$ such that for each reduced decomposition $\left(s_{1}, s_{2}, \ldots, s_{n}\right)$ of $g$, (see [1] for the definition) $V_{g}=\left\{s_{1}, s_{2}, \ldots, s_{n}\right\}$. If $S$ is a subset of $V$ and $g \in \Gamma_{s}$, then $S \supset V_{g}$.

Panel structure.

DEFINITION $1.3[9]$. A panel structure on a topological space $Q$ is a locally finite family of closed subspaces $\left(Q_{v}\right)_{v \in V}$, indexed by some set $V$. The $Q_{v}$ are the panels of $Q$. A space together with a panel structure is called a $V$-panelled space, and we denote it by $\left\{Q,\left(Q_{v}\right)_{v \in V}\right\}$.

For each $x \in Q$, let $V(x)$ denote the set of $v$ in $V$ such that $x \in Q_{v}$. For each subset $S$ of $V$ denote by $Q_{S}$ the set of $x$ in $Q$ such that $V(x)$ contains $S$. The $Q_{S}$ are the faces of $Q$. The formal boundary of $Q$ is defined as: $\partial Q=\bigcup\left\{Q_{v}: v \in V\right\}$. 
Note that $Q_{\varnothing}=Q$, and $Q_{S}=\bigcap_{v \in S} Q_{v}$.

If $S$ is a subset of $V$, we further define $Q_{\sigma(S)}=\bigcup_{v \in S} Q_{v}$.

Observe that if $A$ is a subspace of a $V$-panelled space $\left\{Q,\left(Q_{v}\right)_{v \in V}\right\}$, then $\left\{A,\left(A_{v}\right)_{v \in V}\right\}$ is also a $V$-panelled space, where the panel structure $\left(A_{v}\right)_{v \in V}$ is defined by the equation $A_{v}=A \cap Q_{v}$.

DEFINITION 1.4. A map of panelled spaces $\left\{Q,\left(Q_{v}\right)_{v \in V}\right\}$ and $\left\{Q^{\prime},\left(Q_{w}^{\prime}\right)_{w \in W}\right\}$ is a continuous function $\Phi: Q \rightarrow Q^{\prime}$ such that for each $v \in V$ with $Q_{v} \neq \varnothing$, there exists $w \in W$ such that $Q_{w}^{\prime} \supseteq \Phi\left(Q_{v}\right)$.

A homeomorphism of panelled spaces is an isomorphism in this category.

The universal space.

DEFINITION $1.5[\mathbf{1 7}]$. Let $(\Gamma, V)$ be a Coxeter group and $\left\{Q,\left(Q_{v}\right)_{v \in V}\right\}$ be a $V$-panelled space. The panel structure of $Q$ is called $\Gamma$-finite if $\Gamma_{V(x)}$ is finite for all $x \in Q$. (See 1.3 for the definition of $V(x)$.)

Let $(\Gamma, V)$ be a Coxeter group and $\left\{Q,\left(Q_{v}\right)_{v \in V}\right\}$ be a $V$-panelled space. Give $\Gamma$ the discrete topology. Consider the following equivalence relation on the topological product $\gamma \times Q$ :

$$
(\alpha, x) \sim(\beta, y) \text { if and only if } x=y \text { and } \beta \alpha^{-1} \in \Gamma_{V(x)} .
$$

The universal space $U(\Gamma, Q)$ is defined as the factor space $\Gamma \times Q / \sim$.

Let $\pi: \Gamma \times Q \rightarrow U(\Gamma, Q)$ be a canonical projection defined by

$$
\pi((\alpha, x))=[\alpha, x] .
$$

The action of $\Gamma$ on $\Gamma \times Q$ is defined by $\alpha(\beta, y)=(\alpha \beta, y)$. This action is compatible with the equivalence relation $\sim$. Hence it induces an action of $\Gamma$ on $U(\Gamma, Q)$, namely $\alpha[\beta, x]=[\alpha \beta, x]$.

Note that $Q$ embeds in $U(\Gamma, Q)$ via $x \rightarrow[1, x]$.

Since for each $[1, x] \in U(\Gamma, Q)$, the isotropy group is $\Gamma_{V(x)}$, for each $[g, x] \in$ $U(\Gamma, Q)$, its isotropy group is $g \Gamma_{V(x)} g^{-1}$.

$U(\Gamma, Q)$ is decomposed as a union of the sets of the form $\alpha Q_{S}, V \supseteq S$. These will be called faces. The intersection of any two faces of $U(\Gamma, Q)$ is a third face (or is empty); one sees this immediately from the following lemma.

Lemma 1.6. Let $g \in \Gamma$ and $V_{g}$ be the subset of $V$ defined in Proposition 1.2. Then $g Q_{S} \cap Q_{T}=Q_{S \cup T \cup V_{g}}$.

PROOF. This follows immediately from the definition of $\sim$.

PROPOSITON 1.7 [17, p. 1088]. Let $Y$ be $a \Gamma$ space and $f: Q \rightarrow Y$ be a map such that $v f(x)=f(x)$ for all $v \in V$ and $x \in Q_{v}$. Then there is a unique $\Gamma$-equivariant map $f^{*}: U(\Gamma, Q) \rightarrow Y$ such that $f^{*}([1, x])=f(x)$ for all $x \in Q$.

In particular, letting $Y=U\left(\Gamma, Q^{\prime}\right)$ we see from 1.5 that a map $f: Q \rightarrow Q^{\prime}$ of $V$-panelled spaces defines a $\Gamma$-equivariant map $U(\Gamma, f):(\Gamma, Q) \rightarrow U\left(\Gamma, Q^{\prime}\right)$ with the above functorial properties.

PROPOSITION 1.8 [17, p. 1088]. Suppose $Q$ is a Hausdorff space. Then $\Gamma$ acts properly on $U(\Gamma, Q)$ if and only if the panel structure on $Q$ is $\Gamma$-finite.

Reflection groups. Let $M$ be a connected manifold. A reflection $r: M \rightarrow M$ is a locally smooth involution such that the fixed set $M^{r}$ separates $M$ into exactly two path components. 
DEFINITION 1.9. Suppose that $\Gamma$ is a discrete group acting properly, locally smoothly and effectively on a connected manifold $M$, and that $\Gamma$ is generated by reflections on $M$. Then $\Gamma$ is called a reflection group on $M$.

Let $\Gamma$ be a reflection group on $M$, and let $R$ denote the set of all reflections in $\Gamma$. For each $x \in M$, let $R(x)$ be the set of all $r \in R$ such that $x$ belongs to $M^{r}$. A point $x$ is called nonsingular if $R(x)=\varnothing$. A chamber of $\Gamma$ on $M$ is defined as the closure of a connected component of the set of nonsingular points.

Let $Q$ be a chamber. Denote by $V$ the set of reflections $v$ such that for some $x \in Q, R(x)=\{v\}$. We now define a panel structure on $Q$ as follows: for each $v \in V$, set $Q_{v}=M^{v} \cap Q$.

THEOREM 1.10 [9, p. 301]. Let $\Gamma$ be a reflection group on a manifold $M$. Let $Q$ be a chamber and $V$ be the set of reflections defined above. The following statements are true:

1. $(\Gamma, V)$ is a Coxeter group. In particular, $V$ generates $\Gamma$.

2. For each $v \in V$ and each $g \in \Gamma$ the relation $L(v g) \geq L(g)$ means that $Q$ and $g Q$ are on the same side of the wall $M^{v}$. (See [1] for the definition of $L(g)$.)

3. $\Gamma$ acts freely and transitively on the set of chambers in $M$.

4. If $x, y \in Q$ and $g x=y$ for some $g \in \Gamma$, then $x=y$ and $g \in \Gamma_{V(x)}$.

5. $Q$ is a closed fundamental domain; i.e. if $\pi: M \rightarrow M / \Gamma$ denotes the orbit map then $\left.\pi\right|_{Q}$ is a homeomorphism.

6. The isotropy group at $x \in Q$ is $\Gamma_{V(x)}$.

Theorem 1.10 shows that for $Q$ and $M$ as above, if $f: Q \rightarrow M$ is the inclusion map, then the $\Gamma$-map $f^{*}: U(\Gamma, Q) \rightarrow M$ defined by 1.7 , is a homeomorphism.

Let $C^{n}$ be the standard simplicial cone in $\mathbf{R}^{n}$ defined by the inequalities $x_{i} \geq 0$, $1 \leq i \leq n$. For any $x=\left(x_{1}, \ldots, x_{n}\right) \in C^{n}$ its codimension $C(x)$ is the number of $x_{i}$ that are equal to zero. For each $i$, set $C_{i}=\left\{x \in C^{n} \mid x_{i}=0\right\}$.

DEFINITION 1.11. A $V$-panelled $n$-manifold is a Hausdorff paracompact panelled space $\left\{Q,\left(Q_{v}\right)_{v \in V}\right\}$ in which each point has a neighborhood $N$ and a homeomorphism of panelled spaces from $\left\{N\left(N_{v}\right)_{v \in V}\right)$ to an open set of $\left\{C^{n},\left(C_{i}\right)_{i=1, \ldots, n}\right\}$.

Note that $Q$ is a manifold with boundary and $\partial Q=\bigcup\left\{Q_{v}, v \in V\right\}$. Also each $Q_{v}$ is a manifold with boundary and $Q_{v}$ is a $V-\{v\}$-panelled $n-1$ manifold, i.e., $Q_{v}$ has codimension one in $Q$. Inductively, then if $S$ is a subset of $V, Q_{S}$ is empty or is an $n-|S|$-dimensional $V-S$-panelled manifold; its boundary is $\partial Q_{S}=\bigcup\left\{Q_{T}: T \supset S,|T|=1+|S|\right\}$.

THEOREM 1.12 [9, p. 308]. Let $(\Gamma, V)$ be a Coxeter group and let $Q$ be a connected $V$-panelled manifold with $\Gamma$-finite panel structure. Let $M=U(\Gamma, V)$. Then $M$ is a manifold and $(\Gamma, V)$ is a reflection group on $M$ with fundamental chamber $Q$.

THEOREM 1.13 [17, p. 1092]. Let $\Gamma$ be a discrete linear group generated by a set $V$ of reflections in the faces of a "convex polyhedral cone" $K$ (see [17]). Let $K^{f}=\left\{x \in K: \Gamma_{V(x)}\right.$ is finite $\}$. Then the following statements hold.

1. $\bigcup_{g \in \Gamma} g K$ is a convex cone.

2. $\Gamma$ acts properly on the interior $\Omega$ of this cone.

3. $\Omega \cap K=K^{f}$.

4. The canonical map from $K^{f}$ to $\Omega / \Gamma$ is a homeomorphism.

5. $\Gamma$ is a reflection group on $\Omega$, and $K^{f}$ is a closed chamber for $\Gamma$ on $\Omega$. 
Notice that the above theorem together with Theorem 1.10 implies that each linear Coxeter group is a Coxeter group.

THEOREM $1.14[17$, p. 1105]. Every Coxeter group is isomoprhic to some linear Coxeter group.

THEOREM $1.15[9$, p. 317]. The virtual cohomological dimension of $\Gamma$ is $\leq$ the dimension of $K(\Gamma, V)$.

Serre in $[\mathbf{1 5}]$ proved that if the $\operatorname{Vcd} \Gamma<\infty$, then there is a finite dimensional $\Gamma$-complex $\xi \Gamma$ such that:

1. $\xi \Gamma$ is a proper $\Gamma$-complex, i.e., $\Gamma$ acts cellularly, the stabilizer of each cell fixes that cell pointwise, and each point has finite isotropy group.

2. For each finite subgroup $H$ of $\Gamma$, the fixed set $(\xi \Gamma)^{H}$ is contractible.

Properties 1 and 2 specify the $\Gamma$-homotopy type of the space $\xi \Gamma$, and we will write $\xi \Gamma$ for any finite dimensional $\Gamma$-complex satisfying properties 1 and 2 .

An immediate consequence of Brown's collaring theorem (see [6]) is the following:

THEOREM 1.16. Let $Q$ be an $n$-dimensional compact topological manifold with boundary $\partial Q$. Then $(Q, \partial Q)$ is an NDR-pair (see [19], for the definition of NDRpair).

\section{ChAPTER II. PROPERTIES OF ADMISSIBLE MANIFOLDS}

Let $(\Gamma, V)$ be a Coxeter group and $\left\{Q,\left(Q_{v}\right)_{v \in V}\right\}$ be an $n$-dimensional panelled manifold. We are going to study the $\Gamma$-manifolds $U(\Gamma, Q)$, when $\left\{Q,\left(Q_{v}\right)_{v \in V}\right\}$ has the property that $Q_{S}$ is contractible if $\Gamma_{S}$ is finite or $Q_{S}$ is empty if $\Gamma_{S}$ is infinite.

LEMMA 2.1. Let $(\Gamma, V)$ be a Coxeter group, and let $H$ be a finite subgroup of $\Gamma$. Then there is a subset $J$ of $V$ such that $J$ generates a finite subgroup $\Gamma_{J}$ of $\Gamma$, and some conjugate of $\Gamma_{J}$ contains $H$.

ProOF. By $[\mathbf{1 7}],(\Gamma, V)$ is isomorphic to some linear Coxeter group, and also is a reflection group on $\Omega$. By $[9], \Omega$ is $\Gamma$-homeomorphic to $U\left(\Gamma, C^{f}\right)$. Let $p$ be any element in $\Omega$. Since $\Omega$ is convex $1 /|H| \sum_{h \in H} h p$ is an element of $\Omega$. Let us call it $t y$, where $y \in C^{f}$ and $t \in \Gamma$. It follows from the definition of $U\left(\Gamma, C^{f}\right)$ that the isotropy group of $t y$ is $t \Gamma_{V(y)} t^{-1}$. Since $H$ fixes $t y$, we get $t \Gamma_{V(y)} t^{-1} \supset H$ as required.

Let $(\Gamma, V)$ be a Coxeter group and $Q$ be an $n$-dimensional connected $V$-panelled manifold with $\Gamma$-finite panel structure. By Theorem 1.12, $U(\Gamma, Q)$ is a manifold and $(\Gamma, V)$ is a reflection group on $U(\Gamma, Q)$ with fundamental chamber $Q$. Let $V \supset J$ be such that $\left|\Gamma_{J}\right|<\infty$, and write $G=\Gamma_{J}$. We want to describe, as efficiently as possible, the fixed set $U(\Gamma, Q)^{G}$.

DEFINITION 2.2. A face $g Q_{S}$ of $U(\Gamma, Q)^{G}$ is called a domain of $U(\Gamma, Q)^{G}$ if ${ }^{g} \Gamma_{S}=G$.

Note that if $Q_{S}$ is nonempty, then it is a domain of $U(\Gamma, Q)^{G}$.

LEMMA 2.3. Assume $U(\Gamma, Q)^{G}$ is connected. Then $U(\Gamma, Q)^{G}=\bigcup\left\{w Q_{T}: w Q_{T}\right.$ is a domain of $\left.U(\Gamma, Q)^{G}\right\}$.

ProOF. Each point of $U(\Gamma, Q)^{G}$ lies in some face $w Q_{T}$ such that $w\left(\operatorname{Int} Q_{T}\right)$ is open in $U(\Gamma, Q)^{G}$, where Int $Q_{T}$ denotes the interior of $Q_{T}$. By the theorem of invariance of domains all these open sets have the same dimension. The points of 
$U(\Gamma, Q)^{G}$ having isotropy group $G=\Gamma_{S}$ form an open dense set in $U(\Gamma, Q)^{G}$, so at least one of these points is in each of the $w\left(\operatorname{Int} Q_{T}\right)$ above. And the result follows.

DEFINITION 2.4. Let $(\Gamma, V)$ be a Coxeter group and $Q$ be a compact $n$ dimensional $V$-panelled manifold. We say that $Q$ is an admissible manifold if the following conditions are satisfied:

1. $Q_{S}$ is contractible if $\Gamma_{S}$ is finite.

2. $Q_{S}$ is empty if $\Gamma_{S}$ is infinite.

Proposition 2.5. Let $(\Gamma, V)$ be a Coxeter group. Assume $Q$ is a $V$-panelled admissible manifold. Assume that $Q^{\prime}$ is a $\Gamma$-finite $V$-panelled space and that for each $S$ in $V,\left(Q_{S}^{\prime}, \partial Q_{S}^{\prime}\right)$ is an NDR-pair. Then there exists a $V$-panelled map $f: Q^{\prime} \rightarrow Q$.

ProOF. The map $f: Q^{\prime} \rightarrow Q$ is constructed inductively on all the faces $Q_{J}^{\prime}$, $V \supset J$ such that $\Gamma_{J}$ is a finite group.

Use the fact that $\left(Q_{T}^{\prime}, \partial Q_{T}^{\prime}\right)$ is an NDR pair and the homotopy extension property in order to conclude that $f=f_{\varnothing}: Q^{\prime} \rightarrow Q$ is the required map.

Let $(\Gamma, V)$ be a Coxeter group. Let $\left\{Q,\left(Q_{v}\right)_{v \in V}\right\}$ and $\left\{Q^{\prime},\left(Q_{v}^{\prime}\right)_{v \in V}\right\}$ be $V$ panelled spaces.

DEFINITION 2.6. A map of $V$-panelled spaces $F:\left\{Q \times I,\left(Q_{v} \times I\right)_{v \in V}\right\} \rightarrow$ $\left\{Q^{\prime},\left(Q_{v}^{\prime}\right)_{v \in V}\right\}$ is called a homotopy from $\left.F\right|_{Q \times 0}$ to $\left.F\right|_{Q \times 1}$.

Obviously if $F$ is a homotopy between $f_{0}, f_{1}:\left\{Q,\left(Q_{v}\right)_{v \in V}\right\} \rightarrow\left\{Q^{\prime},\left(Q_{v}^{\prime}\right)_{v \in V}\right\}$, then $U(\Gamma, F)$ provides a $\Gamma$-equivariant homotopy from $U\left(\Gamma, f_{0}\right)$ to $U\left(\Gamma, f_{1}\right)$.

DEFINITION 2.7. Let $(\Gamma, V)$ be a Coxeter group. Let $\left\{Q,\left(Q_{v}\right)_{v \in V}\right\}$ be a $V$ panelled manifold. Two faces $g Q_{S}$ and $w Q_{T}$ of $U(\Gamma, Q)$ are called adjacent if they have the same dimension and their intersection is a nonempty face of codimension one less than each.

The following is a key result.

Proposition 2.8. Let $(\Gamma, V)$ be a right angled Coxeter group. Let $\left\{Q\left(Q_{v}\right)_{v \in V}\right\}$ be a $\Gamma$-finite $V$-panelled manifold. Let $G=\Gamma_{J}$ be a finite subgroup of $\Gamma$, where $V \supset J$. Let $g Q_{K}$ be a domain of $U(\Gamma, G)^{G}$ that is adjacent to $Q_{J}$. Let $T$ be the subset of $V$ defined by $Q_{T}=Q_{J} \cap g Q_{K}$. Then

a. $T=J \cup V_{g}$.

b. $|T|=|J|+1$, and $\Gamma_{T}$ is a finite group and $K=J$. $T$.

c. $g Q_{J}=v Q_{J}$, where $v$ is an element of $V$ that commutes with all elements of

PROOF. a. Follows from 1.6.

b. Follows from the definition of adjacent faces.

c. Follows from the definition of right angled Coxeter group and the fact that $\Gamma_{T}$ and $\Gamma_{J}$ are elementary abelian 2 groups, $\Gamma_{T} / G$ has order 2 and is generated by $g \Gamma_{J}=v \Gamma_{J}$ where $T=J \cup\{v\}$. This completes the proof.

PROPOSITION 2.9. Let $S, T$ be subsets of $V$. Assume that $Q_{S} \cap g Q_{T} \neq \varnothing$ for some $g \in \Gamma$. Let $x \in Q_{S} \cap g Q_{T}$. Then $g \in \Gamma_{V(x)}, V(x) \supset S \cup T$ and $Q_{S} \cap Q_{T} \supset$ $Q_{s} \cap g Q_{T}$.

PrOOF. Follows from the definition of $U(\Gamma, Q)$ and 1.6. 
PROPOSITION 2.10. Let $(\Gamma, V)$ be a right angled Coxeter group and let $\left\{Q,\left(Q_{v}\right)_{v \in V}\right\}$ be an admissible $V$-panelled manifold. Let $G=\Gamma_{J}$ be a finite group, where $J$ is some subset of $V$. Assume that $U(\Gamma, G)^{G}$ is connected. Let $g Q_{J}$ be any domain of $U(\Gamma, Q)^{G}$. Then $g Q_{J}=v_{1} \cdot v_{2} \cdot v_{3} \cdots v_{m} Q_{J}$ for some elements $v_{i} \in V$ that commute with all elements of $J$.

Proof. Since $U(\Gamma, Q)^{G}$ is a connected manifold, there is a finite sequence of domains of $U(\Gamma, Q)^{G}$ of the form $Q_{J}, g_{1} Q_{J}, g_{2} Q_{J}, \ldots, g_{m} Q_{J}=g Q_{J}$, each one adjacent to the next. Apply 2.8 and by induction if follows that $g Q_{J}=v_{1}$. $v_{2} \cdots v_{m} Q_{J}$ and each $v_{i}$ commutes with $J$.

Note that from the above proposition $g=v_{1} \cdot v_{2} \cdots v_{m} \cdot x$ where $v_{i} \in V-J$, $x \in \Gamma_{J}$, and each $v_{i}$ commutes with $x$.

LEMMA 2.11. Let $(\Gamma, V)$ be a Coxeter group. Let $\left\{Q,\left(Q_{v}\right)_{v \in V}\right\}$ be an admissible $V$-panelled manifold. Let $H$ be a finite subgroup of $\Gamma$ such that $U(\Gamma, Q)^{H}$ is connected. Then there is an isotropy group $K$ such that $U(\Gamma, Q)^{H}=U(\Gamma, Q)^{K}$.

PROOF. Each isotropy group $K$ has the form $g \Gamma_{S} g^{-1}$, where $V \supset S$ and $\Gamma_{S}$ is a finite group; hence there are only a countable number of isotropy subgroups.

But $U(\Gamma, Q)^{H}=\bigcup\left\{U(\Gamma, Q)^{K}: K \supset H, K\right.$ an isotropy group $\}$.

If $K \supset H, U(\Gamma, Q)^{K}$ is closed in $U(\Gamma, Q)^{H}$, and is a submanifold of $U(\Gamma, Q)^{H}$. But $U(\Gamma, Q)^{H}$ is connected. Now use the Baire category theorem in order to conclude that for some isotropy group $K \supset H, U(\Gamma, Q)^{K}=U(\Gamma, Q)^{H}$.

Let $(\Gamma, V)$ be a Coxeter group. Let $J$ be a subset of $V$.

Let $J^{*}=\{v \in V-J$ such that $v$ commutes with all elements of $J\}$.

Let $\left\{Q,\left(Q_{v}\right)_{v \in V}\right\}$ be a $V$-panelled space. Each $Q_{J}$ has the structure of $J^{*}$ panelled space. This panel structure is defined by the rule $\left(Q_{J}\right)_{v}=Q_{J \cup\{v\}}$.

Proposition 2.12. Let $(\Gamma, V)$ be a right angled Coxeter group and $\{Q$, $\left.\left(Q_{v}\right)_{v \in V}\right\}$ be an admissible manifold. Then

(a) $U(\Gamma, Q)$ is a $\Gamma$-space of type $\xi \Gamma$.

(b) If $J$ is a subset of $V$ such that the group $G=\Gamma_{J}$ is finite, then the natural map $K: \Gamma_{J^{*}} \rightarrow N_{\Gamma}(G) / G$, sending $g$ to $g G$, is an isomorphism.

(c) The natural map $F: U\left(\Gamma_{J^{*}}, Q_{J}\right) \rightarrow U(\Gamma, Q)^{G}$ sending $[g, x]$ to $[g, x]$, if $g \in \Gamma_{J^{*}}$ and $x \in Q_{J}$, is a $K$-equivariant homeomorphism.

ProOF. (a) Is a consequence of (c) as follows: by [9, Theorem 10.1, part 4], $U\left(\Gamma_{J^{*}}, Q_{J}\right)$ is contractible. Now use 2.11 , in order to conclude that $U(\Gamma, Q)$ has type $\xi \Gamma$.

So we only have to prove (b) and (c).

Now $G=\Gamma_{J}$ is a finite abelian group of order $2^{q}$ for some $q$.

$U(\Gamma, Q)$ is contractible by $[\mathbf{9}$, Theorem 10.1$]$, so $U(\Gamma, Q)^{G}$ is $Z / 2$-acyclic by Smith theory (see [3, III Theorem 5.2]). In particular $U(\Gamma, Q)^{G}$ is connected. By 2.10, each domain of $U(\Gamma, Q)^{G}$ has the form $g Q_{J}$ where $g \in \Gamma_{J^{*}}$. But if $g_{1} \in N_{\Gamma}(G)$, $g_{1} Q_{J}$ is also a domain in $U(\Gamma, Q)^{G}$. So for some $g \in \Gamma_{J^{*}}, g_{1}=g \cdot x$ where $x \in \Gamma_{J}$. It follows that $K$ is an epimorphism. If $g \in \operatorname{ker}(K)$, then $g \in \Gamma_{J^{*}} \cap \Gamma_{J}$. So by 1.2, $J^{*} \cap J \supset V_{g}$. Since $J^{*} \cap J=\varnothing, g=1$. Therefore $K$ is an isomorphism.

It is clear that $F: U\left(\Gamma_{J^{*}}, Q_{J}\right) \rightarrow U(\Gamma, Q)^{G}$ maps $g Q_{J}$ homeomorphically onto ${ }_{g} Q_{J}$ for all $g \in \Gamma_{J^{*}}$ and so yields a homeomorphism from $U\left(\Gamma_{J^{*}}, Q_{J}\right)$ to $U(\Gamma, Q)^{G}$. This completes the proof of 2.12 . 
Proposition 2.13. Let $(\Gamma, V)$ be a right angled Coxeter group. Let $Q$ be an admissible manifold. Let $Q^{\prime}$ be a $V$-panelled manifold with $\Gamma$-finite panel structure. Then any $\Gamma$-map $g: U\left(\Gamma, Q^{\prime}\right) \rightarrow U(\Gamma, Q)$ is $\Gamma$-homotopic to a $\Gamma$-map $U(\Gamma, f)$ : $U\left(\Gamma, Q^{\prime}\right) \rightarrow U(\Gamma, Q)$, where $f$ is a $V$-panelled map from $Q^{\prime}$ to $Q$.

ProOF. By [15] and 1.15, $\operatorname{Vcd} \Gamma<\operatorname{dim} K(\Gamma, V)$. Since $\operatorname{dim} K(\Gamma, V)$ is finite, $\operatorname{Vcd} \Gamma<\infty$.

By $2.12, U(\Gamma, Q)$ is of type $\xi \Gamma$. Since for each subset $S$ of $V$ such that $\Gamma_{S}$ is finite, $Q_{S}^{\prime}$ is a finite dimensional manifold with boundary $\partial Q_{S}^{\prime}$. So by Theorem 1.16, $\left(Q_{S}^{\prime}, \partial Q_{S}^{\prime}\right)$ is an NDR-pair for each subset $S$ of $V$ such that $\Gamma_{S}$ is finite. Use 2.5 , in order to get a $V$-panelled map $f: Q^{\prime} \rightarrow Q$, and extend $f$ to a $\Gamma$-map $U(\Gamma, f): U\left(\Gamma, Q^{\prime}\right) \rightarrow U(\Gamma, Q)$. By $[\mathbf{8}], g$ and $U(\Gamma, f)$ are in the same $\Gamma$-homotopy class.

PROPOSITION 2.14. Suppose that $\Gamma$ is a group generated by a set $V$ of reflections on some manifold $X$, such that $X / \Gamma$ is compact and $X$ is of type $\xi \Gamma$. Let $M$ be any $\Gamma$-manifold of type $\xi \Gamma$ such that $M / \Gamma$ is compact. Then $M^{v}$ has codimension one in $M$ for each $v \in V$.

Proof. By $1.15, \operatorname{Vcd} \Gamma \leq$ the dimension of $K(\Gamma, V)$. So there is a torsion free normal subgroup $G$ of $\Gamma$ of finite index.

Since $X$ and $M$ are of type $\xi \Gamma$, each $G$-isotropy group is trivial. So the projections $P: X \rightarrow X / G$ and $P^{\prime}: M \rightarrow M / G$ are regular covering maps and therefore $X / G$ and $M / G$ are of type $K(G, 1)$, and $H_{*}(G) \approx H_{*}(X / G) \approx H_{*}(M / G)$.

We conclude that $X / G=M / G$, and dimension of $X=$ dimension of $M$. Since $M_{\Gamma} \approx X$, we have $X_{H}^{v} \approx M^{v}$, where $H=Z_{G}(v)$.

By [8], $X^{v} / Z_{G^{(v)}}=(X / G)^{\langle v\rangle}$.

Since $[\Gamma: G]<\infty, X / G$ is closed. But $X / \Gamma$ is compact, so $X / G$ is compact. So $X^{v} / Z_{G^{(v)}}$ is compact. The same holds for $M$.

Use Proposition 7.5 and Corollary 7.6 in [4, p. 209], in order to conclude that $H_{c}^{*}\left(X^{v}: Z\right) \approx H_{c}^{*}\left(Z_{G}(v): Z Z_{G}(v)\right) \approx H_{c}^{*}\left(M^{v}: Z\right)$. Therefore, $M^{v}$ has the same codimension of $X^{v}$ for each $v \in V$.

\section{CHAPTER III. RIGIDITY THEOREMS}

Let $(\Gamma, V)$ be a Coxeter group. Let $M$ and $M^{\prime}$ be admissible manifolds. We are going to prove under some conditions on the group $\Gamma$ and on $M$ and $M^{\prime}$, that $M$ and $M^{\prime}$ are equivariantly homeomorphic.

In this chapter we will assume that the reader is familiar with the concepts in surgery theory as exposed by C. T. C. Wall [18], and W. Browder [3].

LEMMA 3.1. Let $\left(M^{n}, \partial M\right)$ be a compact contractible manifold. Then the structure set $\mathscr{S}\left(M^{n}, \partial M\right)=*$ if $n \neq 3$.

PROOF. Since $M$ is compact contractible, $\partial M$ has the homology of the $n-1$ sphere.

Use the surgery exact sequence, the result $N M(M, \partial)=[M / \partial M, F /$ Top $]=$ $\left[S^{n}, F /\right.$ Top] (see [12]), the fact that $\mathscr{P}\left(S^{n}\right)=*$, by the genralized Poincaré conjecture for $n \geq 5$, and Freedman for $n=4$ (see [11]) in order to conclude that $\mathscr{S}\left(M^{n}, \partial M\right)=*$ if $n \neq 3, n \geq 5$. 
The case when $n=4$, follows from the 5-dimensional $h$-cobordism theorem $[\mathbf{1 3}$, and 12].

DEFINITION 3.2. Let $Q$ be an admissible manifold. We say that $Q$ is a strongly admissible manifold if it satisfies the following condition:

** If $Q_{S}$ is a 3-dimensional panel of $Q$, then $Q_{S}$ is homeomoprhic to $\left.I^{3}\right\}$.

PROPOSITION 3.3. Let $Q$ and $Q^{\prime}$ be strongly admissible manifolds. If $f: Q \rightarrow$ $Q^{\prime}$ is a map of $n$-dimensional compact $V$-paralled manifolds, then there is a homotopy $F_{t}$ of $V$-panelled maps from $F=f$ to a homeomorphism $g$.

Proof. Let $\Psi=\left\{J, J\right.$ subset of $V$ such that $f_{J}: Q_{J} \rightarrow Q_{J}^{\prime}$ is a homeomorphism, where $\left.f_{J}=g \mid Q_{j}\right\}$.

Clearly $\Psi$ is a nonempty set.

Let $J$ be any subset of $V$ such that $J$ satisfies the following conditions:

1. If $K \supset J$ and $K \neq J$, then $K \in \Psi$.

2. $J \notin \Psi$.

It is clear that for any such $J, f \mid \partial Q_{J}: \partial Q_{J} \rightarrow \partial Q_{J}^{\prime}$ is already a homeomorphism.

It is enough to show that there is a homotopy $F_{t}$ of $V$-panelled mapps relative to $\bigcup_{K \in \Psi} Q_{K}$ from $F_{0}=f$ to a map $g$ such that $g \mid Q_{J}: Q_{J} \rightarrow Q_{J}^{\prime}$ is a homeomorphism.

By repeated use of the homotopy extension property it is enough to show that $f \mid Q_{J}: Q_{J} \rightarrow Q_{J}^{\prime}$ is homotopic relative to $\partial Q_{J}$, to a homeomorphism.

If the dimension of $Q_{J}$ is not equal to 3 , the result follows from Lemma 3.1, since $f \mid Q_{J}:\left(Q_{J}, \partial Q_{J}\right) \rightarrow\left(Q_{J}^{\prime}, \partial Q_{J}^{\prime}\right)$ is a homotopy equivalence of pairs.

Suppose that the dimension of $Q_{J}$ is equal to 3 . Since $Q_{J}$, and $Q_{J}^{\prime}$ are homeomorphic to $I^{3}$, we conclude that $f \mid Q_{J}:\left(I^{3}, \partial\right) \rightarrow\left(I^{3}, \partial\right)$ is homotopic relative to $\partial I^{3}$, to a homeomorphism $h$, where $h$ is defined as follows $\left(\left.f\right|_{\partial I^{3}}: S^{2} \rightarrow S^{2}\right.$ is an isomorphism, extend to $h=c\left(\left.f\right|_{\partial I^{3}}\right): I^{3} \rightarrow I^{3}$, and define $c\left(\left.f\right|_{\partial I^{3}}\right)(\alpha v)=\alpha\left(\left(\left.f\right|_{\partial I^{3}}\right)(v)\right)$, $0 \leq \alpha \leq 1)$.

REMARK. If the Poincaré conjecture is true for $n=3$, then admissible manifolds are strongly admissible, so Proposition 3.3, is valid for admissible manifolds.

Proposition 3.4. Let $(\Gamma, V)$ be a right angled Coxeter group. Let $Q$ and $Q^{\prime}$ be strongly admissible manifolds. Any $\Gamma$-map $f_{0}: U(\Gamma, Q) \rightarrow U\left(\Gamma, Q^{\prime}\right)$ is $\Gamma$-homotopic to a $\Gamma$-homeomorphism.

ProOF. By 2.13, we may as well assume $f_{0}$ has the form $U(\Gamma, f)$ where $f: Q \rightarrow Q^{\prime}$ is a map of $V$-panelled spaces. By Proposition 3.3, $f$ is homotopic to a homeomorphism $g: Q \rightarrow Q^{\prime}$ of $V$-panelled manifolds. So $f_{0}$ is $\Gamma$-homotopic to $U(\Gamma, g)$, which is obviously a $\Gamma$-homeomorphism.

DEFINITION 3.5 Let $\Gamma$ be a Coxeter group acting locally smoothly and effectively on a manifold $M$. We say that $M$ is a Coxeter manifold for $\Gamma$, if it satisfies the following conditions:

1. $M / \Gamma$ is compact.

2. $\Gamma$ acts properly discontinuously on $M$.

3. $M^{H}$ is contractible for any finite subgroup $H$ of $\Gamma$.

4. If $M^{H}$ has dimension 3 , then $M^{H} \approx R^{3}$.

REMARK 3.6. In [9], Michael Davis showed that certain Coxeter groups $(\Gamma, V)$ act on contractible manifolds $X^{n}\left(\neq R^{n}, n \geq 4\right)$ with compact quotients. These Davis groups have the following general properties: 
1. The generators $v \in V$ act as reflections on $X$.

2. The action is properly discontinuously, locally smooth and effective.

3 . The only entries of the Coxeter matrix are 1,2 , and $\infty$.

4. If $S$ is a subset of $V$ generating a finite subgroup $G$ of $\Gamma$, then $X^{G}$ is contractible.

Note that all Davis' examples are Coxeter manifolds.

DEFINITION 3.7. A Coxeter group that admits an action with the above properties $1-4$ of 3.6 is called a right angled reflection group.

Proposition 3.8. Let $(\Gamma, V)$ be a right angled reflection group. Let $M$ be a Coxeter manifold for $\Gamma$. Then $M$ is $\Gamma$-homeomoprhic to $U(\Gamma, Q)$, where $Q$ is a fundamental domain.

Proof. By Remark 3.6, $X$ is a Coxeter manifold for $\Gamma$ of type $\xi \Gamma$. By 2.14, $M^{v}$ has codimension one in $M$ for each $v \in V$. We need only show that $M-M^{v}$ has exactly two components.

Assume that $n=\operatorname{dim}(M)$. Taking cohomology with compact support with coefficient in $Z$, the long exact sequence in cohomology is reduced to $0 \rightarrow Z \rightarrow$ $H_{c}^{n}\left(M, M^{v}\right) \rightarrow Z \rightarrow 0$.

So $H_{c}^{n}\left(M, M^{v}\right) \approx Z \oplus Z$. But $H_{c}^{n}\left(M, M^{v}\right) \approx H_{0}\left(M-M^{v}\right)$. Therefore $M-M^{v}$ has exactly two components.

Let $M_{0}=M-\left(\bigcup_{v \in V} M^{v}\right)$. Let $C$ be a connected component of $M_{0}$. Define $Q$ as the closure of $C$ in $M . Q$ is a fundamental domain, by 1.10 , and $\left.\Pi\right|_{Q}$ is a homeomorphism, where $\Pi: M \rightarrow M / \Gamma$. By hypothesis, $M / \Gamma$ is compact, so $Q$ is compact.

The natural panel structure $\left(Q_{v}\right)_{v \in V}$ of $Q$ is defined as follows:

$Q_{v}=Q \cap M^{v}$.

Let $i: Q \rightarrow M$ be the inclusion of its fundamental domain. Then the induced map $i^{*}: U(\Gamma, Q) \rightarrow M$ is a $\Gamma$-equivariant homeomorphism (see [9]). So we conclude that $M \approx U(\Gamma, Q)$.

COROLLARY 3.9. Let $(\Gamma, V)$ be a right angled relfection group. Let $M$ be a Coxeter manifold for $\Gamma$. Then $M$ is $\Gamma$-homeomorphic to $U(\Gamma, Q)$, where $Q$ is an admissible manifold.

ProOF. By 3.8, $M$ is $\Gamma$-homeomorphic to $U(\Gamma, Q)$, where $Q$ is a compact fundamental domain. Let $S$ be a subset of $V$. Write $G=\Gamma_{s}$ a finite subgroup of $\Gamma$. By $2.11, U(\Gamma, Q)^{G}=U\left(\Gamma_{S^{*}}, Q_{S}\right) . U(\Gamma, Q)^{H}$ is contractible by [9, Theorem 10.1] $Q_{S}$ is contractible. Therefore, we conclude that $Q$ is an admissible manifold.

At this point, we have the following conjecture about Coxeter manifolds.

ConjeCture. If $M$ and $M^{\prime}$ are Coxeter manifolds, then $M$ and $M^{\prime}$ are equivariantly homeomorphic.

We are going to prove this conjecture only in the case when the Coxeter group $\Gamma$ is a right angled reflection group.

The following theorem is a key fact for our purpose.

THEOREM 3.10. Let $(\Gamma, V)$ be a right angled reflection group. Let $M$ be a Coxeter manifold. Then $M$ is $\Gamma$-homeomorphic to $U(\Gamma, Q)$, where $Q$ is a compact strongly admissible manifold. 
ProOF. Since Vcd $\Gamma<\infty$ and by hypothesis $M^{H}$ is contractible for each finite subgroup $H$ of $\Gamma$, so by [8], $M$ is of type $\xi \Gamma$. By Corollary $3.9, M$ is $\Gamma$-homeomorphic to $U(\Gamma, Q)$, where $Q$ is a compact admissible manifold.

Let $H$ be any finite subgroup of $\Gamma$ such that the dimension of $M^{H}$ is equal to 3 . By hypothesis $M^{H}=R^{3}$, so $U(\Gamma, Q)^{H} \approx R^{3}$. Using $2.11, U(\Gamma, Q)^{H}=U(\Gamma, Q)^{K}$, where $K$ is an isotropy group, $K \supset H$. Since $K$ has the form $g G g^{-1}$, where $G=\Gamma_{S}$, $V \supset S, g \in \Gamma$, and $\Gamma_{S}$ is a finite subgroup of $\Gamma$; and $U(\Gamma, Q)^{K}=g U(\Gamma, Q)^{G}$, we get that $U(\Gamma, Q)^{K}=g U\left(\Gamma_{S^{*}}, Q_{S}\right)$.

Claim. $Q_{S}$ is homeomorphic to $I^{3}$.

Proof of claim. Since $Q_{S}$ is a compact contractible 3-dimensional manifold with boundary in $R^{3}, \partial Q_{S}$ is a 2-dimensional closed manifold of genus zero. So $\partial Q_{S}=$ $S^{2}$, using the Schonflies theorem [5]. The closures of the components of $S^{3}-S^{2}$ are 3-dimensional balls, so we conclude that $Q_{S}$ is a 3-dimensional ball. Consequently $Q$ is a strongly admissible manifold.

The following is our principal result:

THEOREM 3.11 (RIgIDITY THEOREM FOR COXETER MANIFOLDS). Let $(\Gamma, V)$ be a right angled reflection group. Any two Coxeter manifolds $M$ and $N$ are equivariantly homeomorphic. In fact, any $\Gamma$-map between them is $\Gamma$-homotopic to a homeomorphism.

ProOF. Let $M$ and $M^{\prime}$ be Coxeter manifolds. By 3.11, $M$, (respectively $M^{\prime}$ ) is $\Gamma$-homeomorphic to $U(\Gamma, Q)$, (respectively $U\left(\Gamma, Q^{\prime}\right)$ ), where $Q$ and $Q^{\prime}$ are compact strongly admissible manifolds.

For each subset $S$ of $V$, such that $\Gamma_{S}$ is finite, $Q_{S}$ and $\partial Q_{S}$ are compact manifolds. By Theorem 1.16, $\left(Q_{S}, \partial Q_{S}\right)$ is an NDR-pair. Therefore there is a homotopy equivalence $f: Q \rightarrow Q^{\prime}$ of $V$-panelled spaces. By 2.13, any $\Gamma$-map $g: U(\Gamma, Q) \rightarrow U\left(\Gamma, Q^{\prime}\right)$ is $\Gamma$-homotopic to a $\Gamma$-map $U(\Gamma, f): U(\Gamma, Q) \rightarrow U\left(\Gamma, Q^{\prime}\right)$. By 3.4, $U(\Gamma, f)$ is $\Gamma$-homotopic to a homeomorphism.

\section{BIBLIOGRAPHY}

1. N. Bourbaki, Groupes et algèbres de Lie, Chapitres IV, V, Hermann, Paris, 1968.

2. G. E. Bredon, Introduction to compact transformation groups, Academic Press, New York, 1972.

3. W. Browder, Surgery on simple connected manifolds, Springer-Verlag, New York, 1972.

4. K. S. Brown, Cohomology of groups, Springer-Verlag, New York, 1982.

5. M. Brown, A proof of the generalized Schonfiies theorem, Bull. Amer. Math. Soc. 66 (1960), 74- 76.

6. __ Locally flat embeddings of topological manifolds, Ann. of Math. (2) 75 (1962), 331-341.

7. R. W. Carter, Finite groups of Lie type, conjugacy class and complex characters, Pure and Appl. Math., Ser. of Texts, Monographs \& Tracts, Wiley-Interscience.

8. F. Connolly and T. Kozniewski, Finiteness properties of classifying spaces of proper $\Gamma$-actions, J. Pure Appl. Algebra (to appear).

9. M. W. Davis, Groups generated by reflections and aspherical manifolds not covered by Euclidean space, Ann. of Math. (2) 117 (1983), 293-324.

10. F. T. Farrell and W. C. Hsiang, The topological Euclidean space form problem, Invent. Math. 45 (1978), 181192.

11. M. Freedman, The topology of four dimensional manifolds, J. Differential Geometry 17 (1982), $357-453$.

12. R. Kirby and L. Siebenmann, Foundational essays on topological manifolds, smoothing and triangulations, Ann. of Math. Studies, no. 88, Princeton Univ. Press, Princeton, N.J., 1977.

13. F. Quinn, Ends of maps. III: Dimension 4 and 5, J. Differential Geometry 17 (1982), 503-521. 
14. C. P. Rouke and B. J. Sanderson, Introduction to piecewise linear topology, Springer-Verlag, New York, 1982.

15. J. P. Serre, Cohomologie des groupes discrets, Prospects in Math., Ann. of Math. Studies, no. 70, Princeton Univ. Press, Princeton, N.J., 1971, pp. 77-169.

16. E. H. Spanier, Algebraic topology, McGraw-Hill, New York, 1966.

17. E. B. Vinberg, Discrete linear groups generated by reflections, Math. USSR Izv. 5 (1971), 10831119.

18. C. T. C. Wall, Surgery on compact manifolds, Academic Press, New York, 1970.

19. G. W. Whitehead, Elements of homotopy theory, Springer-Verlag, New York, 1978.

Department of Mathematics, University of Notre Dame, Notre Dame, INDiANA 46556

Current address: Departamento de Matematicas, Núcleo de Sucre, Universidad de Orient, Cumaná, Estado Sucre, Venezuela 